\title{
NAT 7 BN: bosques plantados clonales de nogal híbrido (Juglans $x$ intermedia MJ 209) para la producción sostenible de maderas nobles
}

\author{
NAT 7 BN: Planted forests with clonal hybrid walnut \\ (Juglans $x$ intermedia MJ 209) \\ for sustainable production of precious timber
}

Fernández-Moya, J. ${ }^{*}$; Licea-Moreno, R. ${ }^{1}$; Santacruz, D. ${ }^{1}$; Urbán-Martínez, I. ${ }^{1}$ Bosques Naturales S.A. Avda. De la Vega 1, Alcobendas . 


\title{
Resumen
}

Desde finales del siglo XX se han desarrollado en España diferentes modelos de plantaciones enfocadas a la producción de maderas nobles (nogal-Juglans sp.- y cerezo-Prunus sp.-). El éxito de estas ha sido dispar, considerándose factores como el material genético y la selección de sitio como claves para el éxito/fracaso de estas inversiones. Este trabajo presenta resultados del crecimiento de plantaciones forestales de nogal clonal (NAT-7-BN) en diferentes condiciones edafoclimáticas en varias localidades de España. El NAT-7-BN alcanza una clase de sitio I en Galicia (13 años) un DAP de 19,7 cm, mientras que se considera como clase de sitio I/II en Cuenca ( 8 años) $11,5 \mathrm{~cm}$, en Toledo (13 años) 17,8 cm y en Girona (15 años) 18,5 cm. Las plantaciones procedentes de semilla pueden producir unos resultados aceptables en crecimiento medio (p.ej. DAP de 22,8 cm en 19 años en Cáceres o $21 \mathrm{~cm}$ en 18 años en Girona); sin embargo, estos se producen con una muy elevada variabilidad entre árboles, alternándose ejemplares con 30-40 cm DAP (o incluso más) en 18-19 años y otros con DAP menor a $15 \mathrm{~cm}$.

Palabras clave: clones, maderas nobles, material forestal de reproducción cualificado, nogal, plantaciones forestales.

\begin{abstract}
Since the end of the XX century many forest plantations were established in Spain to produce precious timber (walnut-Juglans sp.- y cherry-Prunus sp.-). The result of these plantations has been variable, and factors such as the genetic material and site selection determine the success or failure of the investments. This paper shows the results of growth from several walnut plantations established with the NAT-7-BN clone in Spain under different edaphoclimatic characteristics. NAT-7-BN reaches a site class I in Galicia, with a DBH of $19.7 \mathrm{~cm}$ in 13 years, while it ranges between site classes I/II in Cuenca (11.5 cm in 8 years), in Toledo (17.8 cm in 13 years) and in Girona (18.5 cm in 15 years). Plantations established with seedlings grown from seeds can also have relatively good average growth rates (DBH of $22.8 \mathrm{~cm}$ in 19 years in Caceres or $21 \mathrm{~cm}$ in 18 years in Girona); however, trees show high variability, with some trees showing DBH of 30-40 cm (or even more) in 18-19 years while others show very low growth. The shape of the trees is also very variable in plantations established using seedlings cultivated from seeds, resulting in many trees with good growth and bad shape, which is a problem for the commercialization and the timber processing.
\end{abstract}

Keywords: clones, forest plantations, precious timber, walnut. 


\section{Introducción}

Desde finales del siglo XX se plantaron en España entre 8.000 y 10.000 ha de plantaciones forestales enfocadas a la producción de maderas nobles (principalmente nogal-Juglans sp.- y cerezo-Prunus sp.-) (Aletà 2014). Sólo considerando los bosques plantados de nogal, en España se han plantado 6.000 ha desde la década de 1990s beneficiándose de diferentes ayudas de programas europeos para la potenciación de la reforestación (Pemán-García et al., 2018), mientras que en otros países europeos se estima que se han plantado alrededor de 60.000 ha, fundamente en Italia y, en menor grado, en Francia (Pelleri et al., 2019). En ese sentido, se engloban dentro de la denominación genérica de plantaciones de nogal (para madera) las correspondientes a diferentes especies, fundamentalmente: nogal común o europeo (Juglans regia L.), nogal negro o americano (J. nigra L., J. major (Torr.) A. Heller, $J$. hindsii (Jeps.) Jeps. ex R.E. Sm.) e híbridos entre ellos especialmente concebidos para la producción de madera (p.ej. Mj-209xRa y Ng-23xRa) (Aletà 2004; Victory et al., 2004; Mohni et al., 2009; Clark y Hemery 2010; Coello et al., 2013).

Estas plantaciones de nogal para la producción de madera de calidad se han establecido principalmente en tierras que anteriormente estaban dedicadas al cultivo agrícola y se han gestionado de manera relativamente intensiva, con densidades iniciales de plantación medio-bajas (250-400 árboles ha ${ }^{-1}$ ) y un turno esperado entre 25 y 50 años (Cisneros et al., 2008; Mohni et al., 2009; Coello et al., 2013; Fernández-Moya et al., 2019). El éxito de este tipo de plantación ha sido dispar. En ese sentido, las plantaciones procedentes de material de semilla presentan una acusada variabilidad a pesar de ser árboles con un alto grado de parentesco (Aletà y Vilanova 2006), considerándose este factor genético (además de la selección de sitio y la gestión selvícola) como uno de los factores principales que determinan el éxito/fracaso de estas inversiones.

Teniendo en cuenta la importancia que se le ha dado a la selección de material genético de nogal para producción de madera, en España ha habido durante estas últimas décadas dos grandes grupos de trabajo:

1. Desde el IRTA (p.ej. Aletà et al. 2003; Aletà 2004).

2. Desde Bosques Naturales SA (p.ej. Urbán-Martínez et al., 2013; Licea-Moreno 2016; Fernández-Moya et al., 2019).

En el Catálogo Nacional de Materiales de Base (CNMB) de España se incluyen cinco clones de Juglans x intermedia Mjx209 (procedentes de la selección de Bosques Naturales SA), siete clones de Juglans nigra, cuatro progenitores de familia de híbridos artificiales y cinco de Juglans regia (MAPA 2018). En ese contexto, desde 2000 la empresa Bosques Naturales SA decidió usar sólo planta clonal en sus plantaciones y cuenta con bosques de nogal clonal plantados en sus fincas de Girona, Cuenca, Toledo y Galicia. Este trabajo presenta los resultados de crecimiento de estas plantaciones forestales de nogal clonal (NAT-7-BN) en diferentes condiciones edafoclimáticas. 


\section{Material y métodos}

\subsection{El clon NAT-7-BN}

El clon NAT-7-BN está catalogado en el CNMB como "CL-Q-751/Eurowalnut B-07" (MAPA 2018) y procede de un árbol-plus seleccionado de la plantación forestal de Bosques Naturales SA en Villanueva de la Vera (Cáceres) según el trabajo de selección temprana de árboles-plus llevado a cabo en 2000 sobre árboles plantados en 1997/1998. La selección de este clon se debe principalmente a su elevado crecimiento juvenil y los buenos resultados que ofrece en las tareas de micropropagación en laboratorio (superiores a otros clones con los que trabaja la empresa).

\subsection{La gestión de las plantaciones de estudio}

Los bosques plantados de nogal que se describen en este trabajo han sido gestionados de forma relativamente homogénea. La densidad de plantación inicial es de 333 plantas ha $^{-1}(5 \times 6 \mathrm{~m}$ ). Los primeros años se realiza un control de malezas utilizando herbicidas y desbroces mecanizados y gradeos y se realizan podas según el crecimiento de las plantaciones hasta una altura de tronco de $4 \mathrm{~m}$ (excepto en Galicia que se consideran $5 \mathrm{~m}$ ). La fertilización y el riego se realizan en general por fertirriego según las características edafoclimáticas diferenciadas de las distintas fincas, excepto en Galicia en las que hay zonas que, debido a la ausencia de riego, la fertilización se realiza en cobertera. La aplicación de dolomita ( 1 t/ha cada dos años hasta los 15 años) también se realiza en cobertera, pero únicamente en Galicia y Cáceres.

\subsection{Descripción de los sitios de estudio}

El presente trabajo se basa en los datos de las plantaciones forestales de Bosques Naturales SA en sus fincas de Galicia, Girona, Cuenca, Toledo y Cáceres, cuyas principales características edafoclimáticas se describen en la Tabla 1.

\subsection{Análisis de datos}

Se utilizan los datos correspondientes al inventario periódico realizado por la empresa Bosques Naturales SA. Este inventario se basa en la medición periódica de una malla sistemática de árboles homogéneamente distribuidos dentro de cada una de las "poblaciones dasométricas". Estas "poblaciones dasométricas" son rodales plantados en el mismo año con plántulas del mismo origen y características (p.ej. mismo clon si son clonales o todas de semilla con el mismo origen en caso de ser procedentes de semilla). El inventario sistemático realizado consiste en la medición de la altura y el diámetro de un promedio de 9 árboles ha ${ }^{-1}$, aunque esta cantidad de árboles muestreados es diferente en cada población dasométrica (Tabla 2). Dentro de la base de datos de la empresa, en este trabajo se muestran los datos 
Tabla 1. Descripción general de la zona de estudio en los bosques plantados de nogal híbrido (Juglans $x$ intermedia Mj209) en distintas regiones de la empresa Bosques Naturales SA (España)

\begin{tabular}{|c|c|c|c|c|c|}
\hline Finca & Galicia & Girona & Cuenca & Toledo & Cáceres \\
\hline Localidades & $\begin{array}{c}\text { Arzúa } \\
\text { y Boimorto } \\
\text { (A Coruña) }\end{array}$ & $\begin{array}{l}\text { Pontós } \\
\text { y Navata } \\
\text { (Girona) }\end{array}$ & $\begin{array}{c}\text { Landete y } \\
\text { Fuentelespino de } \\
\text { Moya (Cuenca) }\end{array}$ & $\begin{array}{c}\text { El Carpio } \\
\text { de Tajo } \\
\text { (Toledo) }\end{array}$ & $\begin{array}{l}\text { Villanueva } \\
\text { de la Vera } \\
\text { (Cáceres) }\end{array}$ \\
\hline $\begin{array}{l}\text { Coordenadas geográficas } \\
\left({ }^{\circ} \text { Lat/Long) }\right.\end{array}$ & $42,98 /-8,19$ & 42,19 / 2,89 & $39,92 /-1,40$ & $39,85 /-4,47$ & $40,11 /-5,37$ \\
\hline $\begin{array}{l}\text { Elevación } \\
\text { (m snm) }\end{array}$ & 435 & 130 & 1030 & 400 & 290 \\
\hline $\begin{array}{l}\text { Precipitación } \\
\text { media anual }(\mathrm{mm})\end{array}$ & 1400 & 700 & 500 & 500 & 1000 \\
\hline Meses secos & 2 & 3 & $3-4$ & 5 & 4 \\
\hline $\begin{array}{l}\text { Temperatura } \\
\text { media }\left({ }^{\circ} \mathrm{C}\right)\end{array}$ & 12 & 14 & 12 & 16 & 16 \\
\hline $\begin{array}{l}\text { Clasificación climática } \\
\text { (Papadakis) }\end{array}$ & $\begin{array}{l}\text { Mediterráneo } \\
\text { templado }\end{array}$ & $\begin{array}{c}\text { Mediterráneo } \\
\text { continental }\end{array}$ & $\begin{array}{l}\text { Mediterráneo } \\
\text { templado }\end{array}$ & $\begin{array}{c}\text { Mediterráneo } \\
\text { subtropical }\end{array}$ & $\begin{array}{c}\text { Mediterránec } \\
\text { subtropical }\end{array}$ \\
\hline Textura & $\begin{array}{c}\text { franco } \\
\text {-arenosa }\end{array}$ & $\begin{array}{l}\text { franco } \\
\text {-limosa }\end{array}$ & $\begin{array}{c}\text { franco } \\
\text {-arenosa }\end{array}$ & $\begin{array}{c}\text { franco-arcillo } \\
\text {-arenosa }\end{array}$ & $\begin{array}{l}\text { arenoso } \\
\text {-franca }\end{array}$ \\
\hline Materia Orgánica (\%) & 7,8 & 1,2 & 0,6 & 1,1 & 2,4 \\
\hline $\mathrm{pH}$ & 5,5 & 8,3 & 8,6 & 7,8 & 5,6 \\
\hline Carbonatos (\%) & & 25 & 12 & & \\
\hline Caliza activa (\%) & & 18 & 5 & & \\
\hline
\end{tabular}

Tabla 2. Detalle de la superficie y el número de árboles inventariados en cada uno de las poblaciones dasométricas de los bosques plantados de nogal híbrido (Juglans x intermedia Mj209) en distintas regiones de la empresa Bosques Naturales SA (España).

\begin{tabular}{|c|c|c|c|c|c|c|c|}
\hline \multicolumn{4}{|c|}{ RODALES CLONALES "NAT-7-BN" } & \multicolumn{4}{|c|}{ RODALES DE SEMILLA } \\
\hline Finca & Población dasométrica & Area (ha) & $\mathrm{n}$ & Finca & Población dasométrica & Area (ha) & $\mathrm{n}$ \\
\hline \multirow[t]{2}{*}{ Galicia } & GaMN2 & 34,78 & 103 & \multirow[t]{3}{*}{ Cáceres } & CaNo1 & 54,19 & 94 \\
\hline & GaMN1 & 12,55 & 18 & & $\mathrm{CaNo} 2$ & 18,29 & 102 \\
\hline \multirow{4}{*}{ Gerona } & GeRo2A & 3.755 & 46 & & CaNo6 & 0,76 & 20 \\
\hline & GeRo2B & 3,755 & 26 & \multirow[t]{2}{*}{ Gerona } & GeRo1 & 35,68 & 121 \\
\hline & GeRo3 & 4,39 & 30 & & GeRo5 & 0,43 & 19 \\
\hline & GeRo4 & 1,81 & 31 & & & & \\
\hline \multirow[t]{4}{*}{ Cuenca } & CuN7A 07 & 11,85 & 23 & & & & \\
\hline & CuN7B 07 & 11,56 & 10 & & & & \\
\hline & CuN7A & 5,79 & 74 & & & & \\
\hline & CuN7B & 0,79 & 4 & & & & \\
\hline \multirow[t]{3}{*}{ Toledo } & ToSN7a & 11,38 & 62 & & & & \\
\hline & ToSN7b & 11,08 & 32 & & & & \\
\hline & ToSN7c & 1,41 & 9 & & & & \\
\hline
\end{tabular}


de los rodales establecidos con material clonal de NAT-7-BN, así como otros procedentes de semilla que se utilizan para comparar (Tabla 2). Para cada una de estas poblaciones dasométricas se calcula la media y el intervalo de confianza (con un 95\% de significación) de la variable en cuestión.

Los datos de estas poblaciones dasométricas se comparan con los modelos de clases de sitio propuestos por Montero y Cisneros (2006) (expuestos en Cisneros et al., 2008), asignándoles una clase que posibilita una mejor comparación entre los rodales de distinta edad y una mejor discusión de los datos. En este trabajo se realiza una modificación a dicha propuesta, considerándose una calidad adicional intermedia entre Calidad I y Calidad II ("Calidad I/II").

\section{Resultados y discusión}

El clon NAT-7-BN presenta en Galicia (13 años) un comportamiento muy homogéneo en las dos poblaciones dasométricas analizadas, alcanzando un DAP de $20,8 \mathrm{~cm}( \pm 0,3$ CI95\%), 19,9 cm ( $\pm 0,8$ CI95\%) y correspondiéndose con un sitio de Calidad I (Figura 1). En Toledo (12 años) también tiene un comportamiento muy homogéneo en los tres rodales analizados, con un DAP de 16,5 cm $( \pm 0,3$ CI95\%), $16,5 \mathrm{~cm}( \pm 0,4 \mathrm{CI} 95 \%)$ y $15,1 \mathrm{~cm}( \pm 1,1 \mathrm{CI} 95 \%)$, correspondiéndose con sitios de calidad intermedia entre I y II (Calidad I/Calidad II) (Figura 1). En Cuenca tiene un comportamiento más variable en función de las características edáficas de los distintos rodales analizados llegando a tener en los sitios mejores un DAP de 10,23 $\mathrm{cm}( \pm 0,6$ CI95\%) con 7 años (Calidad I), en los sitios intermedios un DAP de 9,26 $\mathrm{cm}( \pm 1,4$ CI95\%) con 7 años y 15,9 cm $( \pm 0,4$ CI95\%) con 13 años (Calidad I/Calidad II) y en los sitios peores un DAP de 13,7 cm ( $\pm 0,8$ CI95\%) con 13 años (Calidad II) (Figura 1). En Girona también presenta un comportamiento variable en función de las características edáficas de los distintos rodales analizados: sitios de Calidad I/Calidad II con un DAP de 15,5 cm ( $\pm 0,9$ CI95\%) con 12 años y 18,5 cm $( \pm 0,7$ CI95\%) con 14 años; y sitios de Calidad II con un DAP de $16,0 \mathrm{~cm}( \pm 1,1$ CI95\%) y 17,3 cm ( $\pm 0,7$ CI95\%), ambas con 15 años (Figura 1).

Como comparación con las poblaciones dasométricas de bosques plantados de nogal con material genético clonal NAT-7-BN, las plantaciones procedentes de material producido a partir de semilla pueden también producir unos resultados de crecimiento medio que se clasifiquen como Calidad I o como Calidad II (Figura 1). Así, en uno de los rodales muestreados en Cáceres las condiciones edáficas favorables permitieron alcanzar la Calidad I con $25,4 \mathrm{~cm}( \pm 1,5 \mathrm{CI} 95 \%)$ en 18 años mientras que en dos rodales con fertilidad más moderada los nogales alcanzan un DAP de 17,8 cm ( $\pm 0,5$ CI95\%) con 18 años y 18,8 cm ( $\pm 0,7$ CI95\%) con 19 años (Calidad II) (Figura 1). Los rodales procedentes de material de semilla en Girona también son variables en función de la fertilidad de los sitios: un rodal de Calidad I/Calidad II con un DAP de $12,7 \mathrm{~cm}( \pm 1,2 \mathrm{CI} 95 \%)$ con 10 años y un rodal de Calidad II con un DAP de 15,9 cm ( $\pm 0,5$ CI95\%) en 10 años (Figura 1). Sin embargo, estos crecimientos medios se producen con una muy elevada variabilidad entre 
los árboles, alternándose ejemplares con DAP entre 30 y $40 \mathrm{~cm}$ (o incluso más) en 18-19 años y otros con un crecimiento muy pobre.

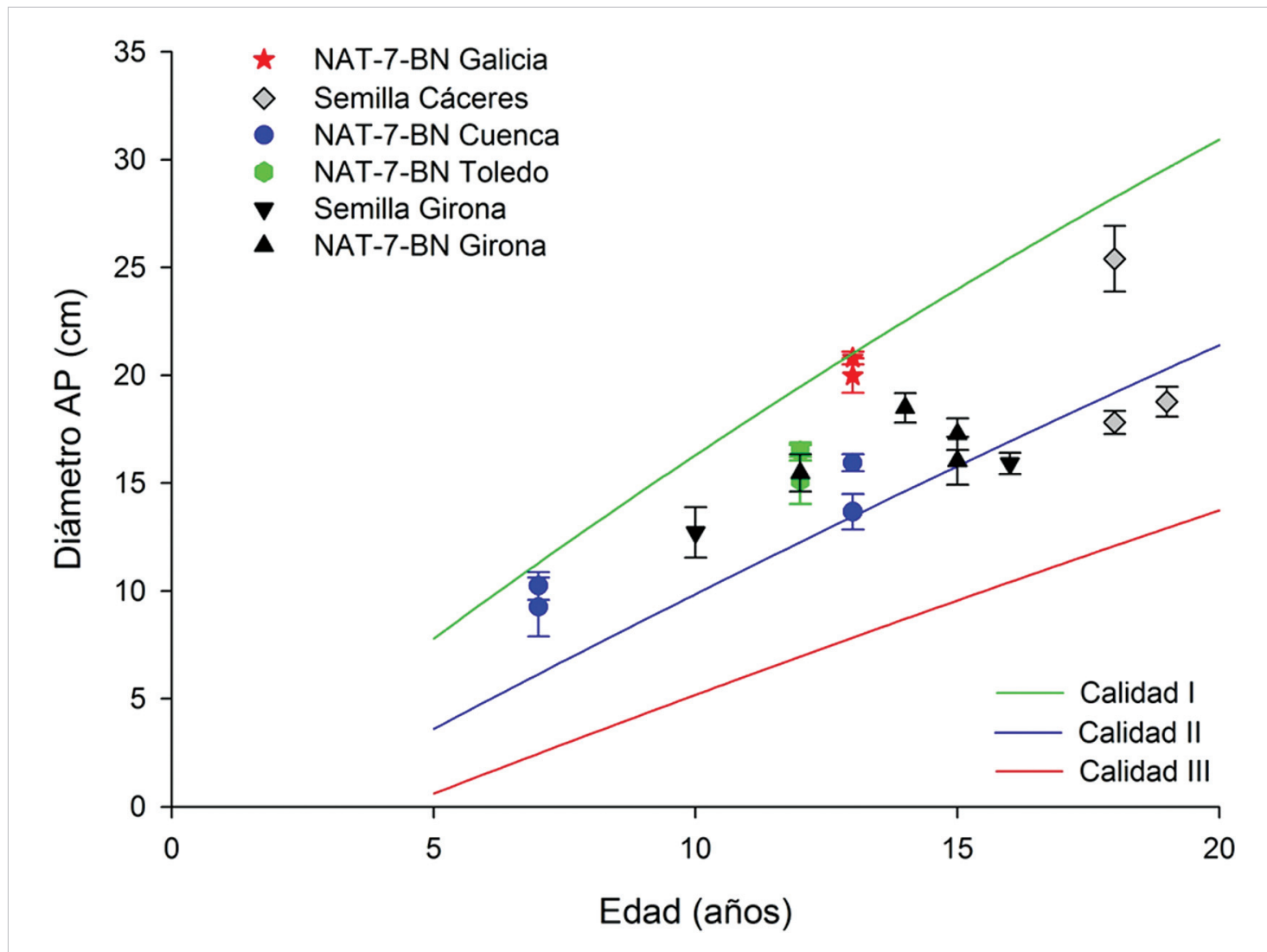

Figura 1. Diámetro normal $(\mathrm{cm})$ en relación con la edad de los bosques plantados de nogal híbrido (Juglans $x$ intermedia Mj209) en distintas regiones de la empresa Bosques Naturales SA (España). Los datos mostrados (medias e intervalos de confianza al 95\% de probabilidad) representan plantaciones establecidas con material genético del clon NAT-7-BN en Galicia, Cuenca, Toledo y Girona y con material genético de semilla en Cáceres y Girona. Las líneas representan las curvas de calidad de sitio para plantaciones de nogal elaboradas por Montero y Cisneros (2006).

La forma del árbol y la rectitud del tronco ha sido tradicionalmente un aspecto especialmente relevante en la selección de clones para la producción de madera (Ehrenberg 1970; Callister et al., 2011; Blackburn et al., 2013) y ha sido identificado como especialmente importante en especies de nogal, que tiene una tendencia generalizada a troncos curvados (Woeste y McKenna 2004; Aletà y Vilanova 2011). En ese sentido, esta característica es considerada como la principal ventaja que motiva el uso de material clonal en los bosques plantados para producción de madera de calidad por parte de Bosques Naturales SA. Así, los bosques plantados de nogal híbrido (Juglans x intermedia Mj209) establecidos a partir de semillas presentan resultados variables en cuanto a forma y rectitud del árbol (Figura 2), mientras que las plantaciones clonales presentan resultados homogéneos (Figura 2), 

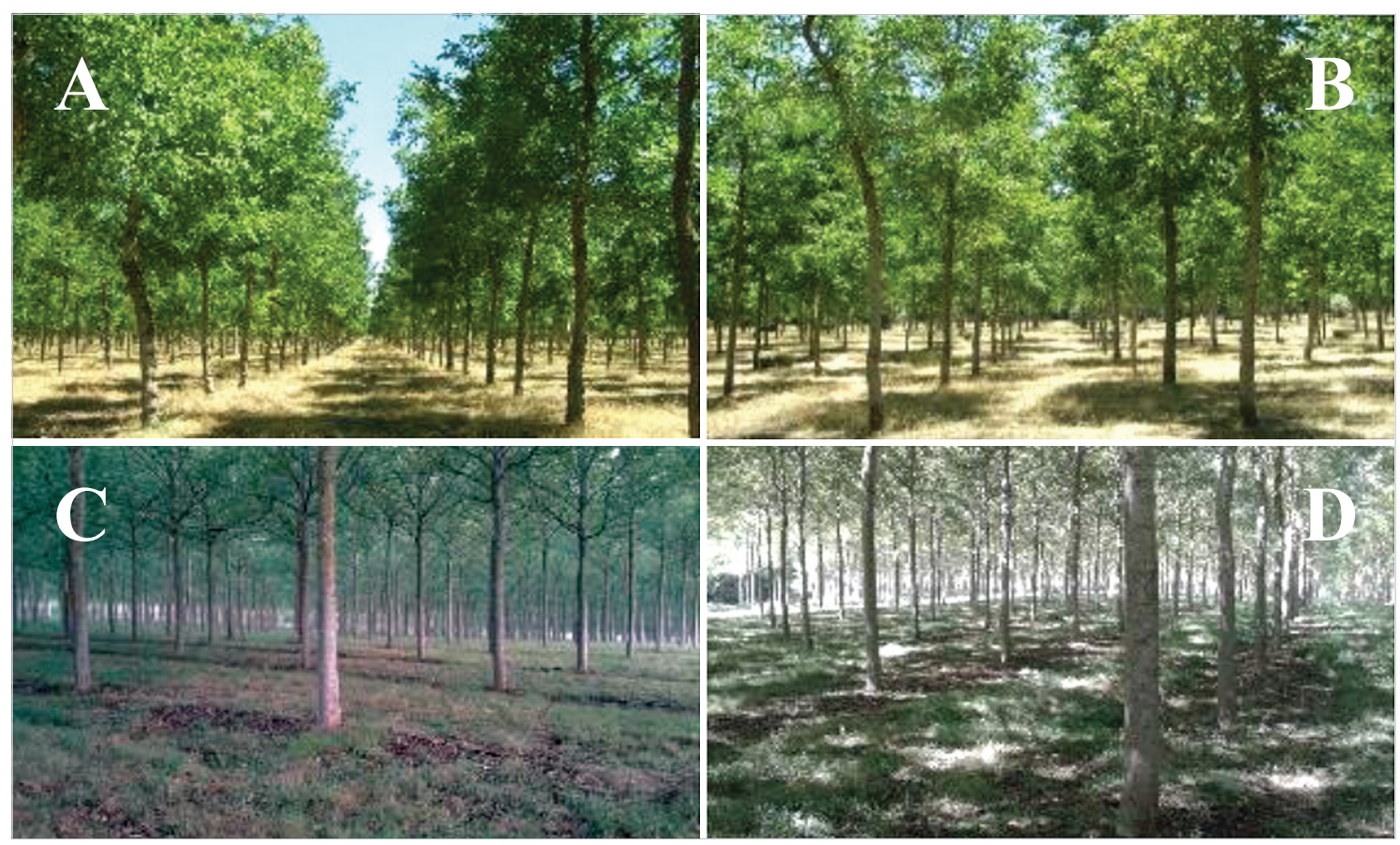

Figura 2. Fotos que muestran la variabilidad y las diferencias en la forma de los árboles en bosques plantados de nogal híbrido (Juglans x intermedia Mj209) establecidos a partir de semillas (A y B) y la homogeneidad y la buena forma en general de los clones establecidos (C y D). Todas las fotos se corresponden con bosques plantados de la empresa Bosques Naturales SA en España de la misma especie y con material genético del mismo origen. Todo el material de semilla procede del mismo vivero y el material clonal procede de una selección propia de la empresa a partir del material de semilla.

teniendo en cuenta una comparación de la misma especie con el mismo material genético del mismo origen.

La homogeneidad entre los árboles es la otra gran ventaja que supone el uso de material clonal en este tipo de bosques plantados (Licea-Moreno 2016). Teniendo en cuenta que estas plantaciones suponen una inversión relativamente alta (suelos fértiles y gestión intensiva, incluyendo en algunos casos el riego), depender de la variabilidad genética de la semilla para obtener o no un rendimiento adecuado añade una componente de riesgo a la inversión que dificulta la misma. Además, la homogeneidad es un aspecto importante para la comercialización de la madera. La forma de los árboles es igual de variable que el crecimiento de los mismos o incluso más, presentándose algunos árboles con buen crecimiento y mala forma, lo que hace que luego no se puedan aprovechar convenientemente por la industria de la madera. En ese sentido, la homogeneidad en la forma también aumenta mucho la eficiencia en los trabajos de poda. En definitiva, el uso de clones asegura una homogeneidad en las plantaciones (disminuyen así el riesgo en la inversión) y busca un equilibrio entre un crecimiento alto de los árboles y un fuste recto que optimice su aprovechamiento posterior por la industria. 
En base a estas características anteriormente descritas, el uso de material clonal supone una cierta garantía de que las plantas que se establecen en campo van a presentar una buena tasa de crecimiento (dependiendo de las características edafoclimáticas del sitio) y, sobre todo, un comportamiento homogéneo y una buena rectitud del fuste. Estas garantías son especialmente relevantes cuando se reduce la densidad inicial de establecimiento (p.ej. 150 plantas ha $^{-1}$ a densidad definitiva), teniendo en cuenta que en estos casos no se plantea la realización de clareos juveniles ni de claras no comerciales y que si éstas se realizan deberían poder tener un aprovechamiento comercial. Esta tendencia está muy relacionada con el uso de nogales para la producción de madera de calidad integrado en sistemas agroforestales, según se está promoviendo desde hace algunos años por diversos autores (p.ej. Eichhorn et al., 2006; Rigueiro-Rodríguez et al., 2009; Coello et al., 2015, 2018; Urbán-Martínez et al., 2018; Vilanova et al., 2018).

NAT-7-BN es el nombre comercial del clon catalogado en el CNMB como “CL-Q-751/Eurowalnut B-07” (MAPA 2018) y ha sido clave en el desarrollo de los bosques plantados de nogal de la empresa Bosques Naturales durante los últimos 15 años. Ripoll et al. (2017) analizaron el comportamiento de diferentes clones de Juglans $x$ intermedia Mjx209 en 3 ensayos clonales replicados en Galicia, Toledo y Granada. En este trabajo, en el que se muestra el desarrollo juvenil (hasta 4 años) de diferentes clones, se observa como el clon NAT-7-BN presenta crecimientos medios comparados con el resto, destacando el crecimiento (tanto en altura como en diámetro) de otros clones como DA o DN (también obtenidos por Bosques Naturales SA). Estos clones DA y DN fueron también incluidos en la selección temprana realizada en el año 2000 en la que se seleccionó el NAT-7-BN, la cual se realizó en base al crecimiento juvenil y fueron posteriormente seleccionados de nuevo como árboles plus en el trabajo realizado por Urbán-Martínez et al. (2013). Como muestran los resultados expuestos anteriormente, el clon NAT7-BN presenta una muy buena capacidad de adaptación de sus tasas de crecimiento en las distintas condiciones edafoclimáticas en las que se ha plantado (Figura 1) y un fuste recto que permite un buen aprovechamiento de la madera (Figura 2). En ese sentido, las buenas propiedades mostradas y el equilibrio entre ellas, unido a que presenta una mayor eficiencia en su micropropagación in-vitro comparada con otros clones (Licea-Moreno 2016), ha hecho que el clon NAT-7-BN haya sido extensamente plantado por la empresa. En cuanto a las propiedades de la madera del clon NAT-7-BN, Merlo-Sánchez et al. (2013) observaron cómo ésta presenta buenas propiedades tanto físicas como estéticas, así como una buena forma general del tronco.

\section{Conclusiones}

NAT-7-BN es el nombre comercial del clon catalogado en el CNMB como "CLQ-751/Eurowalnut B-07”, registrado por la empresa Bosques Naturales SA. Los resultados muestran que el clon presenta una muy buena capacidad de adaptación de 
sus tasas de crecimiento en las distintas condiciones edafoclimáticas en las que se ha plantado: desde Calidad I (hasta $21 \mathrm{~cm}$ de DAP en 13 años) en los mejores sitios hasta Calidad II (desde $17 \mathrm{~cm}$ de DAP en 15 años) en los peores sitios. Además de buenas tasas de crecimiento, el clon NAT-7-BN presenta un fuste recto que permite un buen aprovechamiento de la madera, la cual presenta buenas propiedades físicas y estéticas. Las buenas propiedades mostradas y el equilibrio entre ellas, unido a que presenta una mayor eficiencia en su micropropagación in-vitro comparada con otros clones, ha hecho que el clon NAT-7-BN haya sido extensamente plantado por la empresa.

\section{Agradecimientos}

Los resultados de este trabajo se enmarcan dentro del proyecto europeo WOODNAT (www.woodnat.eu), financiado por la Comisión Europea en el marco del programa H2020. Los autores quieren expresar su agradecimiento explícito al resto de sus compañeros de la empresa Bosques Naturales SA quienes han colaborado en el diseño, establecimiento y seguimiento de estas plantaciones durante estos años. Además, la empresa Bosques Naturales agradece la Ayuda Torres Quevedo que recibe para la contratación de Jesús Fernández Moya, co-autor de este trabajo.

\section{Bibliografía}

Aletà, N.; 2004. Current Research in Spain on Walnut for Wood Production. In: Michler, C.H.; Pijut, P.M.; Van Sambeek, J.W.; Coggeshall, M.V.; Seifert, J.; Woeste, K.; Overton, R.; Ponder, F.Jr.; (eds.), Black walnut in a new century, proceedings of the 6th Walnut Council research symposium. U.S. Department of Agriculture, Forest Service, North Central Research Station, Lafayette, pp. 153-155.

Aletà, N.; Ninot, A.; Voltas, J.; 2003. Caracterización del comportamiento agroforestal de doce geneotipos de nogal (Juglans sp.) en dos localidades de Cataluña. Invest. Agrar.: Sist. Recur. For. 12(1), 39-50

Aletà, N.; Vilanova, A.; 2006. El nogal híbrido. Navarra Forestal 13:18-21

Aletà, N.; Vilanova, A.; 2011. Criterios Orientadores para la Admisión de Materiales de Base del Género Juglans. Ministerio de Medio Ambiente y Medio Rural y Marino. 39 p.

Aletà, N.; 2014. Using walnut species for timber production in Southern Europe. ISHS Acta Horticulturae 1050: 383-388. DOI: 10.17660/ActaHortic.2014.1050.53

Blackburn, D.P.; Hamilton, M.G.; Harwood, C.E.; Baker, T.G.; Potts, B.M.; 2013. Assessing genetic variation to improve stem straightness in Eucalyptus globulus. Annals of Forest Science 70: 461-470. https://doi.org/10.1007/s13595-013-0277-9

Callister, A.N.; England, N.; Collins, S.; 2011. Genetic analysis of Eucalyptus globulus diameter, straightness, branch size, and forking in Western Australia. Canadian Journal of Forest Research 41(6): 1333-1343. https://doi.org/10.1139/x11-036

Cisneros, O.; Montero, G.; Aletà, N.; 2008. Selvicultura de Juglans regia L. In: Serrada, R.; 
Montero, G.; Reque, J.A.; (eds.), Compendio de Selvicultura Aplicada en España. Ed. INIA, Madrid, pp. 207-227

Clark, J.; Hemery, G.; 2010. Walnut hybrids in the UK: fast growing quality hardwoods. Quarterly Journal of Forestry 104: 43-46

Coello, J.; Becquey, J.; Gonin, P.; Ortisset, J.P.; Desombre, V.; Baiges. T.; Piqué, M.; 2013. Guía de ecología y selvicultura de especies productoras de madera de calidad. Generalidad de Cataluña - Centro de la Propiedad Forestal

Coello, J.; Baiges, T.; Cervera, T.; Alcoverro, F.; 2015. Plantacions agroforestals de noguera i freixe amb cultius herbacis. Un sistema productiu innovador amb interès ambiental. In: Tusell, J.M., Vericat, P. (eds.), XXXII Jornades Tècniques Silvícoles Emili Garolera. Consorci Forestal de Catalunya. Santa Coloma de Farners, pp. 60-71.

Coello, J.; Urban-Martínez, I.; Mosquera-Losada, M.R.; 2018. Los sistemas silvoarables modernos en España. Cuad. Soc. Esp. Cienc. For. 44 (2): 19-38. https://doi.org/10.31 167/csefv0i44.17550

Ehrenberg, C.; 1970. Breeding for stem quality. Unasylva Vol. 24 No. 2-3 (97-98) pp.23-31

Eichhorn, M.P.; Paris, P.; Herzog, F.; Incoll, L.D.; Liagre, F.; Mantzanas, K.; Mayus, M.; Moreno, G., Papanastasis, V.P., Pilbeam, D.J., Pisanelli, A., Dupraz, C., 2006. Silvoarable systems in Europe - past, present and future prospects. Agroforestry Systems 67: 29-50. https://doi.org/10.1007/s10457-005-1111-7

Fernández-Moya, J.; Urbán-Martínez, I.; Pelleri, F.; Castro, G.; Bergante, S.; Giorcelli, A.; Gennaro, M.; Licea-Moreno, R.J.; Santacruz-Pérez, D.; Gutiérrez-Tejón, E.; HomarSánchez, C.; Bidini, C.; Chiarabaglio, P.M.; Manetti, M.C.; Plutino, M.; Sansone, D.; 2019. Silvicultural guide to managing walnut plantations for timber production. ISBN 978-84-09-12163-2. Pp 76

Licea-Moreno, R.J.; 2016. Biotecnología forestal aplicada a la producción de madera de nogal. Tesis Doctoral. Universidad Politécnica de Madrid

MAPA, 2018. Catálogo Nacional de Materiales de Base para producción de material forestal de reproducción cualificado: género Juglans. Ministerio de Agricultura, Pesca y Alimentación, Gobierno de España. www.mapa.gob.es/es/desarrollo-rural/temas/poli tica-forestal/juglanssp_clones_tcm30-139313.pdf

Merlo-Sánchez, E.; Santaclara-Estévez, O.; Urbán-Martínez, I.; 2013. Crecimiento, forma y calidad de madera en clones de cerezo y nogal. Estudio clonal y ambiental y uso de métodos no destructivos para su evaluación. VI Congreso Forestal Español, VitoriaGasteiz

Mohni, C.; Pelleri, F.; Hemery, G.E.; 2009. The modern silviculture of Juglans regia L.: A literature review. Die Bodenkultur 60 (3): 21-34

Montero, G.; Cisneros, O.; 2006. Informe sobre el desarrollo del proyecto AGL2003-09347CO2-01, «Selvicultura de plantaciones de frondosas autóctonas productoras de madera de calidad en Castilla y León». Documento de trabajo de uso interno

Pelleri, F.; Castro, G.; Marchi, M.; Fernandez-Moya, J.; Chiarabaglio, P.M.; Giorcelli, A.; Gennaro, M.; Bergante, S.; Manetti, M.; Plutino, M.; Bidini, C.; Sansone, D.; UrbanMartínez, I.; 2019. The walnut plantations between Italy and Spain: the experiences of Woodnat project. Annals of Silvicultural Research [in review]

Pemán-García, J.; Fernández-Centeno, G.; Robla, E.; Lerner, M.; 2018. Balance de un instrumento para el cambio del uso de la tierra: la forestación de tierras agrícolas. In: Oliet-Palá, J.A.; Martínez-Ruiz, C.; Pequeño-Ledezma, M.A.; (eds.) Libro de resúme- 
nes de la IV Reunión conjunta de los grupos de trabajo de repoblaciones forestales (SECF) y de restauración ecológica (AEET). Pp. 41

Rigueiro-Rodríguez, A.; Mc Adam, J.; Mosquera-Losada, M.R.; 2009. Agroforestry systems in Europe: productive, ecologicaland social perspective. Ed. Springer. 450 p.

Ripoll, M.A.; Sánchez-Miranda, A.; Gálvez, C.R.; Navarro, F.B.; Jiménez, M.N.; Homar, C.A.; Urbán, I.; Voltas, J.; Licea-Moreno, R.J.; 2017. Resultados Preliminares de Ensayos Clonales de Nogal Híbrido Maderero. VII Congreso Forestal Español, Plasencia

Urbán-Martínez, I.; Ricardo Licea, R.J.; González, C.; Villar de Arcos, F.; Santaclara, O.; Merlo-Sánchez, E.; 2013. Characterization of walnut hybrid plus trees for good quality wood plantations. International Scientific Conference on Hardwood Processing (ISC HP2013), Florencia

Urbán-Martínez, I.; Fernández-Moya, J.; Licea-Moreno, R.; Santacruz, D.; Gutiérrez-Tejón, E.; 2018. Hybrid walnut (Juglans Mj209) for timber production in an agroforestry scheme: some experiences learnt in Spain. In: Ferreiro-Domínguez, N., Mosquera-Losada, M.R.; (eds.), Proceedings of the 4th European Agroforestry Conference: Agroforestry as Sustainable Land Use. Ed. European Agroforestry Federation and the University of Santiago de Compostela in Lugo (Spain). Pp. 30-34

Victory, E.R.; Woeste, K.E.; Rhodes, O.E.; 2004. History of Black Walnut Genetics Research in North America. In: Michler, C.H.; Pijut, P.M.; Van Sambeek, J.W.; Coggeshall, M.V; Seifert, J.; Woeste, K.; Overton, R.; Ponder, F.Jr.; (eds.), Black walnut in a new century, proceedings of the 6th Walnut Council research symposium. U.S. Department of Agriculture, Forest Service, North Central Research Station, Lafayette, pp. 1-8.

Vilanova, A.; Garcia, D.; Abelló, L.; Rovira, M.; Aletà, N.; 2018. Balance de una producción combinada: madera de nogal y avellana. Cuad. Soc. Esp. Cienc. For. 44(2): 107-120. https://doi.org/10.31167/csefv0i44.17558

Woeste, K.E.; McKenna, J.R.; 2004. Walnut genetic improvement at the start of a new century. In: Michler, C.H.; Pijut, P.M.; Van Sambeek, J.W.; Coggeshall, M.V.; Seifert, J.; Woeste, K.; Overton, R.; Ponder, F.Jr.; (eds.), Black walnut in a new century, proceedings of the 6th Walnut Council research symposium. U.S. Department of Agriculture, Forest Service, North Central Research Station, Lafayette, pp. 9-17. 